\title{
ORGANIZAÇÃO DO TRABALHO DE UMA EQUIPE DE SAÚDE NO ATENDIMENTO AO USUÁRIO EM SITUAÇÕES DE URGÊNCIA E EMERGÊNCIA $^{1}$
}

\author{
Estela Regina Garlet², Maria Alice Dias da Silva Lima, José Luís Guedes dos Santos ${ }^{4}$, Giselda Quintana Marques ${ }^{5}$
}

${ }^{1}$ Artigo extraído da dissertação “O Processo de Trabalho da Equipe de Saúde de uma Unidade Hospitalar de Atendimento às Urgências e Emergências” apresentada ao Programa de Pós-Graduação em Enfermagem (PPGenf) da Universidade Federal do Rio Grande do Sul (UFRGS), 2008.

${ }^{2}$ Mestre em Enfermagem. Enfermeira da Secretaria Municipal de Saúde de Pinhal Grande. Rio Grande do Sul, Brasil. E-mail: estelagarlet@hotmail.com

${ }^{3}$ Doutora em Enfermagem. Professor Associado da Escola de Enfermagem e Docente Permanente do PPGenf/UFRGS. Rio Grande do Sul, Brasil. E-mail: malice@enf.ufrgs.br

${ }^{4}$ Mestrando em Enfermagem do PPGenf/UFRGS. Bolsista Capes. Rio Grande do Sul, Brasil. E-mail: joseenfermagem@yahoo. com.br

${ }^{5}$ Doutoranda em Enfermagem do PPGenf/UFRGS. Enfermeira da Secretaria Municipal de Saúde de Porto Alegre. Rio Grande do Sul, Brasil. E-mail: giseldamarques@hotmail.com

RESUMO: Este artigo teve como objetivo analisar a organização do trabalho da equipe de saúde de uma unidade hospitalar de atendimento a usuários em situações de urgência e emergência do interior do Rio Grande do Sul. Trata-se de um estudo qualitativo, do tipo estudo de caso. Os dados foram coletados por meio de observação e entrevista semi-estruturada com profissionais da equipe de saúde entre junho e setembro de 2007. A análise dos dados seguiu diretrizes do método qualitativo: ordenação, classificação em estruturas de relevância, síntese, interpretação. Constatou-se que o trabalho da equipe divide-se entre atendimento aos casos com potencial risco à vida, assistência aos pacientes na sala de observação e atenção aos usuários com demandas não urgentes. Assim, os profissionais sobrecarregam-se e os pacientes nem sempre têm suas necessidades atendidas adequadamente. O estudo oferece subsídios para reorganização dos processos de trabalho visando à qualidade e resolutividade do atendimento às urgências.

DESCRITORES: Emergências. Serviços médicos de emergência. Equipe de assistência ao paciente. Organização e administração.

\section{WORK ORGANIZATION OF A HEALTH TEAM IN ATTENDING THE USER IN URGENCY AND EMERGENCY SITUATIONS}

\begin{abstract}
The objective of this paper is to analyze the organization of a health team while rendering services in urgency and emergency situations in a hospital unit in the state of Rio Grande do Sul, Brazil. It was a qualitative case study. The data was collected through observation and semi-structured interviews with health professionals between June and September of 2007. The data analysis followed a qualitative approach: ordering, classification by relevance, synthesis, and interpretation. Teamwork is divided into death-risk cases, assistance to patients under observation, and attention to users with non-emergency cases. Thus, professionals are overloaded and patients quite often are not rendered the right service. The study offers support for reorganizing work processes, aiming to improve the quality and effectiveness of emergency service.
\end{abstract}

DESCRIPTORS: Emergencies. Emergency medical services. Patient care team. Organization and administration.

\section{LA ORGANIZACIÓN DEL TRABAJO DE UN EQUIPO DE SALUD EN LA ATENCIÓN AL USUARIO EN SITUACIONES DE URGENCIA Y EMERGENCIA}

RESUMEN: Este trabajo tuvo como objetivo analizar la organización del trabajo de un equipo de salud de una unidad de urgencia y emergencia ubicada en Rio Grande do Sul (Brasil). Se trata de un estudio cualitativo de tipo estudio de caso. La recolección de los datos se hizo por medio de observación y entrevista semiestructurada con profesionales del equipo entre junio y septiembre del 2007. Se realizó un análisis de los datos según las directrices del método cualitativo: ordenación, clasificación por relevancia, síntesis e interpretación. El trabajo del equipo se divide entre la atención a los casos que presentan riesgo de vida, la asistencia a los pacientes en observación y la atención a los usuarios con demandas no urgentes. Así, los profesionales se ven sobrecargados y los pacientes no son atendidos adecuadamente. El estudio ofrece aportes para la reordenación de los procesos de trabajo, tratando de alcanzar la resolutividad de la atención a las urgencias.

DESCRIPTORES: Urgencias médicas. Servicios médicos de urgencia. Grupo de atención al paciente. Organización y administración. 


\section{INTRODUÇÃO}

Nos últimos anos, o sistema brasileiro de atenção às urgências tem apresentando avanços em relação à definição de conceitos e incorporação de novas tecnologias visando à organização do atendimento em rede. Nesse sentido, espera-se que a população acometida por agravos agudos seja acolhida em qualquer nível de atenção do sistema de saúde, de modo que tanto a atenção básica quanto os serviços especializados deverão estar preparados para o acolhimento e encaminhamento de pacientes para os demais níveis do sistema quando esgotarem-se as possibilidades de complexidade de cada serviço.

No entanto, a atenção às urgências tem ocorrido, predominantemente, nos serviços hospitalares e nas unidades de pronto atendimento abertos 24 horas. Esses serviços respondem por situações que vão desde àquelas de sua estrita responsabilidade, bem como um volume considerável de ocorrências não urgentes que poderiam ser atendidas em estruturas de menor complexidade. ${ }^{1}$ Essas situações podem ser identificadas na maioria das unidades públicas de urgência do Brasil e, têm interferido consideravelmente no processo de trabalho e na qualidade do cuidado prestado à população.

Estudo realizado na Emergência de Adultos de um hospital geral de Pernambuco, constatou que $74,5 \%$ dos atendimentos realizados eram por queixas típicas da atenção básica, não se caracterizando, portanto, como urgência. Essa demanda prejudica a assistência aos casos graves e agudos, pois acarreta acúmulo de tarefas, contribui para o aumento dos custos de atendimento e gera sobrecarga para os profissionais da equipe de saúde. ${ }^{2}$

Somado a isso, os profissionais também atendem a situações de extrema gravidade que extrapolam a capacidade resolutiva dos serviços e têm dificuldades para referenciar os pacientes para outros hospitais. Desse modo, as salas de observação, que se destinam à permanência temporária dos pacientes, transformem-se em áreas de internação, sem, no entanto, possuírem as devidas condições de infraestrutura e de pessoal para cuidados contínuos, expondo, com frequência, os pacientes a riscos. ${ }^{3}$

Diante desse contexto, os usuários procuram os serviços hospitalares de urgência como uma das alternativas de acesso, pois entendem que eles reúnem um somatório de recursos, como consultas, remédios, procedimentos de enfermagem, exames laboratoriais e internações, que os tornam mais resolutivos. $^{4}$
Sabe-se que o trabalho em saúde caracterizase pelo encontro entre pessoas que trazem um sofrimento ou necessidades de saúde e outras que dispõem de conhecimentos específicos ou instrumentos que podem solucionar o problema apresentado. Nesse encontro, são mobilizados sentimentos, emoções e identificações que podem dificultar ou facilitar a aplicação dos conhecimentos do profissional na percepção das necessidades ou interpretação das demandas trazidas pelo usuário. Dessa maneira, o cuidado, que é o produto final do trabalho na saúde, é indissociável do processo que o produziu, ou seja, é a própria realização da atividade, sendo consumido pelo usuário no mesmo momento em que é produzido. ${ }^{5}$

A organização do trabalho, entretanto, pode interferir no produto final do trabalho em saúde, transformando-o conforme a influência dos diferentes elementos do processo, das concepções e intenções dos agentes a respeito do produto a ser construído. A organização tecnológica do trabalho se constitui pelos seus elementos: o objeto de trabalho, os instrumentos e a própria atividade, assim como as relações técnicas, sociais e de produção. ${ }^{6}$

Com base no acima descrito, este artigo, elaborado a partir de uma dissertação de Mestra$\mathrm{do}^{7}$, tem como objetivo analisar a organização do trabalho de uma equipe de saúde de uma unidade hospitalar de atendimento a usuários em situações de urgência e emergência do interior do Rio Grande do Sul.

\section{METODOLOGIA}

Trata-se de uma pesquisa qualitativa que apreende a realidade de forma direta e permite a descrição ampla do fenômeno investigado no seu contexto. ${ }^{8} \mathrm{O}$ desenho metodológico da pesquisa é o estudo de caso, o qual permite um aprofundamento da unidade a ser estudada, vista na sua singularidade. ${ }^{9}$

O espaço de pesquisa foi a Unidade de Emergência (EU) de um Hospital de Ensino Público, localizada em um município do interior do estado do Rio Grande do Sul. O serviço atende os usuários que procuram a unidade espontaneamente, e os encaminhamentos formais e informais de unidades pré-hospitalares fixas (Unidades Básicas de Saúde, Unidades de Saúde da Família, Ambulatórios de Especialidades, por exemplo) do município sede e de unidades hospitalares das demais cidades da região, destacando-se como uma referência no atendimento às urgências e emergências. 
Os sujeitos da pesquisa foram selecionados entre os profissionais que atuavam na equipe de saúde da referida unidade, no período de junho a setembro de 2007 , conforme a função que exerciam, buscando abarcar os diversos trabalhos desenvolvidos na unidade. Participaram do estudo 29 sujeitos, sendo sete enfermeiros, nove técnicos de enfermagem, um bolsista do Curso de Enfermagem, dois médicos plantonistas da clínica médica, um médico residente, dois doutorandos do Curso de Medicina, uma secretária, uma auxiliar de serviços gerais, dois vigilantes, uma nutricionista, uma fisioterapeuta e a enfermeira coordenadora administrativa da unidade.

A coleta de dados foi realizada por meio de observação e entrevista semi-estruturada. Os aspectos norteadores da observação foram o objeto, os agentes, os instrumentos e as relações que compunham o processo de trabalho da equipe de saúde que atuava na unidade. Foram observados os diferentes profissionais em atividades consideradas relevantes para captar as relações entre os sujeitos e a forma de organização do trabalho.

As entrevistas semi-estruturadas foram realizadas para identificar as concepções sobre a organização do trabalho, por meio das seguintes questões norteadoras - Como você entende o trabalho realizado na unidade de emergência? Como está organizado o atendimento às urgências e emergências nessa unidade? Quais os profissionais da equipe de emergência que se envolvem no processo de trabalho, e de que forma?

A análise dos dados foi realizada seguindo diretrizes do método qualitativo: ordenação, classificação em estruturas de relevância, síntese e interpretação. ${ }^{8} \mathrm{Na}$ fase de ordenação, realizou-se a digitação dos dados coletados nas observações e entrevistas, a releitura do material e a ordenação dos relatos. A associação entre o material coletado e o referencial teórico ocorreu na etapa de classificação dos dados, a partir de leitura exaustiva e repetitiva dos textos, visando à apreensão das estruturas de relevância. Na etapa de análise final, o material empírico e o teórico foram articulados de forma a obter a interpretação dos fatos. Para organização e operacionalização do processo de análise foi empregado o software Ethnograph versão 5.0 - Qualis Research Associates.

O projeto foi aprovado pelo Comitê de Ética em Pesquisa da Universidade Federal do Rio Grande do Sul sob o No 2007688. Aos profissionais foi entregue um Termo de Consentimento Livre e Esclarecido, o qual garantia os aspectos éticos apon- tados pela resolução N $N^{\circ}$ 196/96 do Conselho Nacional de Saúde. ${ }^{10}$ As informações obtidas por meio das observações e entrevistas foram codificadas com a letra "O" e com a letra " $E$ ", respectivamente, seguidas da descrição da categoria profissional do sujeito observado ou entrevistado.

\section{RESULTADOS E DISCUSSÃO}

Na UE, constatou-se que a organização do trabalho da equipe de saúde é dividida em três momentos: o atendimento às urgências e emergências em que o risco à vida é iminente, a assistência aos pacientes em sala de observação e a atenção aos usuários com demandas de saúde não urgentes.

$\mathrm{O}$ atendimento aos casos graves, com potencial risco à vida é sempre priorizado. Eles têm acesso livre e são encaminhados de forma imediata até a sala de emergência. Nesse momento, salienta-se que os profissionais da equipe de saúde convergem suas ações para o restabelecimento da vida:

[...] quando chega uma emergência é muito rápido, cada um já sabe o que fazer, eu, por exemplo, conheço o olhar da enfermeira quando tem que fazer alguma coisa, é tudo automático, verifica-se os sinais rapidamente, corta-se a roupa rápido, punciona-se a veia rápido, outro já está vendo vias aéreas, estou há quatro anos com o mesmo grupo, a gente já sabe mais ou menos o que cada uma vai pedir (E - Técnica de Enfermagem).

A fala do entrevistado remete à atuação em equipe e à articulação entre as diferentes categorias profissionais no atendimento das situações de urgência e emergência. Esse achado corrobora os resultados de um estudo que destaca a importância da integração e articulação das diferentes categorias profissionais e ramos do conhecimento em prol de uma assistência oportuna e livre de riscos, pautada na troca, cooperação e respostas imediatas ao usuário nas unidades de emergência hospitalares. ${ }^{11}$ Entende-se por articulação as situações de trabalho em que os profissionais colocam em evidência as conexões entre as diversas intervenções executadas e os diversos saberes técnicos empregados na execução do trabalho em saúde. A articulação das ações multiprofissionais e a cooperação são estabelecidas pela comunicação, por meio da mediação simbólica da linguagem. ${ }^{12}$

A interação entre os profissionais no atendimento aos pacientes que tem sua vida ameaçada por uma situação de urgência e emergência pode estar associada ao sentido que eles atribuem ao seu trabalho. A ação de salvar vidas é compre- 
endida como a principal finalidade do trabalho nesta unidade, constituindo-se como um desafio a ser superado pelos profissionais, que se sentem orgulhosos do trabalho que realizam no atendimento desses casos. ${ }^{13}$

Os pacientes graves, após o primeiro atendimento na UE, permanecem em observação, que é o período de tempo transcorrido entre o término da avaliação inicial e a internação em uma unidade aberta ou fechada ou a alta hospitalar. A dificuldade para obter leitos de internação, prolonga o tempo de permanência do paciente na sala de observação, o qual pode se estender por vários turnos ou dias. Tal situação é referida pelos profissionais como uma das dificuldades encontradas no trabalho, visto que muitos desses pacientes são tratados até a alta na nesta unidade.

O paciente acaba sendo tratado, totalmente aqui, sai direto daqui e vai com a alta pra casa. [...] Há pacientes que ficam 30 dias aqui e deveria ser no máximo 24 horas por que aqui é uma unidade de emergência (E - Técnica de Enfermagem).

Segundo a opinião dessa trabalhadora, a unidade que deveria ser transitória, onde o paciente permaneceria um curto período de tempo, passa a funcionar como uma unidade de internação. Isso de certa forma descaracteriza a finalidade do trabalho na emergência e interfere na sua realização, impedindo que seja realizado com a qualidade esperada e não dispondo das condições necessárias para as práticas do cuidado.

Entre a maioria dos trabalhadores e gestores, predomina a concepção de que as unidades hospitalares de atenção às urgências possuem caráter transitório, restrito à estabilização das condições clínicas dos pacientes e o transporte para unidades de cuidados intensivos. A fragmentação e descontinuidade do atendimento fazem com que se perca a visão integral do cuidado, visto que a resolutividade obtida na sala de emergência pode ser perdida, se na observação e/ ou nas unidades de internação, o paciente não tiver um cuidado de qualidade.

Quanto ao atendimento na sala de observação, evidenciou-se que a sua efetivação ocorre de acordo com a gravidade dos pacientes, os quais demandam cuidados médicos e de enfermagem de alta complexidade, pois são, muitas vezes, pacientes em respiração mecânica, que chegam à unidade em decorrência de politraumas, doenças crônicas ou neoplásicas em fase terminal.

Nesse sentido, o atendimento às necessidades básicas do ser humano como sono, repouso, alimentação, higiene corporal e direito à privacidade ficam comprometidos pela excessiva demanda de atendimento, assim como pela proximidade dos leitos. As limitações do ambiente na sala de observação da UE, associada à longa permanência, submetem usuários a constrangimentos físicos e morais:

A equipe de enfermagem, muitas vezes, tem que parar todo o serviço dos pacientes que estão aqui internados ou em observação para atender à emergência, e dai fica praticamente tudo descoberto, [...] fica complicado, tem-se que dividir toda a equipe fica bem mais dificil o atendimento, falta espaço, porque só há uma sala de emergência e se chegam duas emergências é preciso atender às duas juntas na mesma sala (E - Médico).

Essa diversidade de tarefas gera sobrecarga de trabalho entre os profissionais, visto que é complexo realizar múltiplas atividades ao mesmo tempo, especialmente, tratando-se do cuidado a pacientes em estado grave. $\mathrm{O}$ atendimento nas salas de urgência e emergência compete com o atendimento de rotina dos pacientes em observação, como a equipe médica e de enfermagem é a mesma.

Diante desse cenário, mesmo intensificando o ritmo de trabalho, muitas atividades não são realizadas, pois a capacidade de atendimento não é suficiente para atender a demanda na unidade. Segundo enfermeiras e médicos, para que se garantisse um atendimento mais qualificado, $\mathrm{o}$ número de profissionais deveria ser proporcional ao número de pacientes atendidos.

O número de funcionários é pouco, tinha que ser assim, aumenta o número de pacientes, aumenta o número de funcionários (...), a gente atende dentro das possibilidades (E - Enfermeira).

São poucos funcionários para atender tanta gente, tem que ter amor à camiseta para trabalhar aqui (E - Médico).

O enfrentamento das adversidades tem colocado os profissionais na posição de vítimas neste cenário adverso. Para sobreviver ao caos, esquecem que tanto trabalhadores como usuários necessitam condições dignas de trabalho e de assistência.

Estudos apontam que as condições de infraestrutura para o trabalho com espaço físico inadequado para a realização das atividades assistenciais, dimensionamento insuficiente do quadro de profissionais de enfermagem, propiciam tensões e conflitos que se manifestam de forma intensa e estressante sobre os profissionais da unidade, mais especificamente sobre a enfer- 
magem. Assim, a deficiência de recursos humanos e materiais configuram-se como condições impróprias para o trabalho e ameaça à saúde dos trabalhadores que atuam nos serviços hospitalares de emergência. ${ }^{11,13}$

Os profissionais mencionam que a ausência de equipamentos, materiais e a insuficiência de área física são aspectos que contribuem para dificultar a realização do trabalho.

Geralmente, falta material e a gente fica no improviso. O oxímetro, às vezes, está quebrado (E - Técnica de Enfermagem).

Algumas vezes, é difícil conseguir uma maca ou uma cadeira, quando chega paciente aqui. A equipe enfrenta muitos problemas, muitos! Falta maca, falta cadeira (E - Vigilante).

A falta de recursos humanos e materiais, embora não seja constante, foi apontada por todos os profissionais entrevistados e também identificada durante a observação do campo. Essa deficiência dificulta não só o atendimento como também as relações entre trabalhadores e usuários, que demonstram esta insatisfação.

Cabe destacar que essas dificuldades são encontradas na maioria das unidades de urgências e emergências do país. Elas são visivelmente expressas por meio da estrutura física e tecnológica inadequada, insuficiência de equipamentos, recursos humanos limitados, capacitação insuficiente para o trabalho, baixa cobertura do atendimento préhospitalar móvel, número insuficiente de unidades de pronto atendimento e insuficiente retaguarda para transferência de doentes. ${ }^{14}$

Além do atendimento aos casos em que o risco à vida é iminente e da atenção aos pacientes que permanecem em observação, a equipe da unidade divide-se ainda na atenção aos pacientes que necessitam de consulta médica, procedimento de enfermagem, exames e medicações. A demanda por esse tipo de atendimento, além de excessiva, não se esgota no que se considera um problema de saúde. Ela é caracterizada, muitas vezes, por pacientes com necessidades não urgentes, que buscam no atendimento de saúde nas UEs a resolução para os mais diversos problemas sociais e de saúde.

$\mathrm{Na}$ opinião dos profissionais, a procura excessiva por atendimento está relacionada à concentração de recursos humanos e tecnológicos que a UE oferece.

É um hospital-escola, então, às vezes, se consegue uma tomografia imediatamente ou fazer exames comple- mentares. O que o diferencia de outros hospitais, porque eu já trabalhei em hospitais privados e para conseguir uma tomografia, para conseguir um RX, primeiro passa por uma burocracia para saber se o paciente tem convênio, se pode pagar, do contrário, não fazem, aqui o serviço é público e possibilita realizar exames especializados, complementares, cirurgia, enfim todo tratamento (E - Enfermeira).

Os pacientes em situações não urgentes procuram a unidade porque ela está aparentemente mais disponível, pois eles não têm outros recursos para recorrer. Pesquisa realizada em um serviço público de urgência pediátrica evidenciou a heterogeneidade dos atendimentos, contrariando a vocação do serviço, idealizado como referência para casos agudos. O volume excessivo de atendimentos e a percepção da diminuição da qualidade do cuidado prestado foram queixas manifestadas por pediatras do ambulatório de urgências. Os médicos ressentiam-se de assistir crianças que deveriam estar sendo atendidas no cuidado primário, expressando preocupação com as mais graves que também chegavam necessitando de cuidados que não estão disponíveis em outros níveis do sistema. ${ }^{15}$

A escolha do paciente sobre qual unidade deverá acessar pode estar relacionada ao acolhimento anterior de suas demandas por um profissional ou serviço de saúde. Portanto, as concepções e experiências anteriores do paciente relacionadas ao acesso e resolutividade dos serviços de saúde são fatores que podem colaborar com a superlotação e interferem na organização do trabalho da equipe de saúde das unidades hospitalares.

Diante disso, é importante salientar que os significados de urgência para os profissionais e para a população são distintos, como também são diferentes os conceitos de doença e saúde em suas definições biomédicas e nas representações e práticas, que variam segundo segmentos e culturas da sociedade. ${ }^{3}$ Para os profissionais, as urgências estão relacionadas às patologias que comprometem a vida ou função vital importante. Para a população, relacionam-se a necessidades variadas (aflição, angústia, abandono e miséria), que requerem ajuda e/ou assistência, com solução imediata a uma dificuldade passageira. ${ }^{16,17}$

O cenário descrito mostra contradições em relação ao conceito de que a todos os pacientes será garantido o acolhimento nos serviços, de acordo com a complexidade tecnológica, que deverá estar organizada de forma regionalizada, hierarquizada e regulada, pois existem muitas fragilidades na 
organização. Idealmente, os diferentes níveis de atenção devem formar uma rede assistencial de modo que cada serviço complemente a ação de outro por meio de mecanismos organizados e pactuados. Isso só ocorrerá se os serviços se reconhecerem como parte integrante desse sistema de urgências, atendendo adequadamente o paciente naquilo que corresponder a sua capacidade resolutiva.

Portanto, as unidades e os serviços de saúde devem estar habilitados tecnicamente e possuir condições de infra-estrutura física, de pessoal, recursos materiais e equipamentos para prestarem o primeiro atendimento ou a estabilização dos quadros de urgência, e, posteriormente, encaminhar o paciente para unidades de maior porte.

\section{CONSIDERAÇÕES FINAIS}

A análise da organização do trabalho de uma unidade hospitalar de atendimento a usuários em situações de urgência e emergência evidenciou três momentos principais de atuação da equipe de saúde. Os profissionais atendem urgências e emergências em que o risco à vida é iminente, prestam assistência aos pacientes na sala de observação e recebem pacientes com demandas de saúde não urgentes.

A procura por atendimento nos serviços de urgência hospitalar tem inúmeras causas que podem estas associadas ao aumento da violência, das questões socioeconômicas, assim como pela falta de resolutividade de ações e serviços. Os profissionais são interpelados por uma demanda que ultrapassa o que os serviços estão organizados para reconhecer e intervir.

Constatou-se que os profissionais sustentam a concepção biomédica de organização do trabalho e priorizam o atendimento aos usuários com problemas graves e agudos que procuram a unidade e, demonstram insatisfação com o longo tempo de permanência dos pacientes e com a frequente utilização do serviço por casos não urgentes.

A crise dos serviços de urgência pode estar relacionada aos desequilíbrios do sistema de saúde, sendo que os serviços de urgência e emergência se transformam em porta de entrada para os mais variados problemas de saúde e, portanto, sua avaliação permite o acompanhamento da evolução e adequação das políticas implementadas em relação às necessidades da população. Nesse sentido, um atributo de qualidade importante do sistema de saúde na atenção às urgências é a acessibilidade, aliado ao tempo de espera para o atendimento e ao reconhecimento imediato da gravidade dos casos pelos profissionais.

Os resultados desta investigação assinalam de forma relevante a necessidade de qualificação da atenção às urgências. $\mathrm{O}$ estudo oferece subsídios para reorganização dos processos de trabalho e reestruturação dos fluxos de pacientes para as demais unidades do hospital, visando maior resolutividade aos atendimentos e satisfação para os profissionais.

Uma das estratégias possíveis para qualificar o atendimento é a estruturação do acolhimento com avaliação de risco, estabelecendo um equilíbrio entre a demanda de pacientes e os recursos disponíveis para atender suas necessidades, por meio da classificação dos casos.

Aponta-se a necessidade da organização do trabalho na UE com base nos pressupostos da Política Nacional da Atenção as Urgências, que preconizam o acesso e acolhimento aos serviços de saúde de acordo com sua complexidade tecnológica, procedendo a reordenação, quando necessário, a serviços ambulatoriais básicos ou especializados existentes na rede de atenção à saúde. Para tanto, é necessário conhecer a estrutura dos serviços e estabelecer a rede de atenção às urgências, com grades de referência e contra-referência efetivamente pactuadas, com definição de co-responsabilidades, de modo a corrigir as distorções ainda existentes nas portas de entrada do sistema.

\section{REFERÊNCIAS}

1. Deslandes SF, Minayo MCS, Oliveira AF. Análise da implementação do atendimento pré-hospitalar. In: Minayo MCS, Deslandes SF, organizadores. Análise diagnóstica da política de saúde para redução de acidentes e violências. Rio de Janeiro (RJ): Fiocruz; 2007. p.139-57.

2. Furtado BMASM, Araújo Jr. JLC, Cavalcanti P. O perfil da emergência do hospital da restauração: uma análise dos possíveis impactos após a municipalização dos serviços de saúde. Rev Bras Epidemiol. 2004 Set; 7(3):279-89.

3. Giglio-Jacquemot A. Urgências e emergências em saúde: perspectivas de profissionais e usuários. Rio de Janeiro (RJ): Fiocruz; 2005.

4. Marques GQ, Lima MADS. Demandas de usuários a um serviço de pronto atendimento e seu acolhimento ao sistema de saúde. Rev Latino-am Enfermagem. 2007 Jan-Fev; 15(1):13-9.

5. Merhy EE. Saúde: a cartografia do trabalho vivo. São Paulo (SP): Hucitec; 2002.

6. Mendes Gonçalves RB. Tecnologia e organização social das práticas de saúde: características 
tecnológicas do processo de trabalho na rede estadual de centros de saúde de São Paulo. São Paulo (SP): Hucitec; 1994.

7. Garlet ER. O processo de trabalho da equipe de saúde de uma unidade hospitalar de atendimento às urgências e emergências. [dissertação]. Porto Alegre (RS): Universidade Federal do Rio Grande do Sul. Programa de Pós-Graduação em Enfermagem; 2008.

8. Minayo MCS. O desafio do conhecimento: pesquisa qualitativa em saúde. $10^{\mathrm{a}}$ ed. Rio de Janeiro/São Paulo (RJ/SP): Abrasco/Hucitec; 2007.

9. Stake RE. Investigación com estúdio de casos. Madrid (SP): Morata; 1998.

10. Brasil. Ministério da Saúde. Conselho Nacional de Saúde. Resolução 196, de 10 de outubro de 1996. Dispõe sobre as diretrizes e as normas regulamentadoras de pesquisa envolvendo seres humanos. Brasília; 1996.

11. Alves M, Ramos FRS, Penna CMM. O Trabalho interdisciplinar - aproximações possíveis na visão de enfermeiras de uma unidade de emergência. Texto Contexto Enferm. 2005 Jul-Set; 14(3):323-31.
12. Peduzzi M. Equipe multiprofissional de saúde: conceito e tipologia. Rev Saúde Pública. 2001 Fev; 35(1):103-9.

13. Dal Pai D, Lautert L. O trabalho em urgência e emergência e a relação com a saúde das profissionais de enfermagem. Rev Latino-am Enfermagem. 2008 Maio-Jun; 16(3):439-44.

14. Ministério da Saúde (BR). Política nacional de atenção às urgências. $3^{\mathrm{a}}$ ed. Brasília(DF): Ministério da Saúde; 2006.

15. Melo EMC, Assunção AA, Ferreira RA. O trabalho dos pediatras em um serviço público de urgências: fatores intervenientes no atendimento. Cad Saúde Pública 2007 Dez; 23(12):3000-10.

16. Martinez-Almoyna M, Nitschke CAS, organizadores. Regulação médica dos serviços de atendimento médico de urgência: SAMU [online]. 1999 [acesso 2007 Nov 16]. Disponível em: http://neu.saude. sc.gov.br/arquivos/manual_de_regulacao_medica_ de_urgencia.pdf.

17. Barrier G. Les appels d'urgence au Samu Emergency calls in the french Samu system. C R Acad Sci. 2001 Jul; 324(7):663-6. 